J Pediatr. 2014 October ; 165(4): 750-754. doi:10.1016/j.jpeds.2014.06.052.

\title{
Sleep duration and obesity among adolescents transitioning to adulthood: Do results differ by sex?
}

\author{
Shakira F Suglia, ScD ${ }^{1}$, Seema Kara, MPH ${ }^{1}$, and Whitney Robinson, PhD $^{2,3}$ \\ ${ }^{1}$ Department of Epidemiology, Mailman School of Public Health, Columbia University, New York \\ NY \\ 2Department of Epidemiology, University of North Carolina, Chapel Hill, NC \\ ${ }^{3}$ Carolina Population Center, University of North Carolina, Chapel Hill, NC
}

\begin{abstract}
Objectives-To examine the association between short sleep duration and obesity among adolescents (mean age 16 years) transitioning into young adulthood (mean age 21 years) in the National Longitudinal Study of Adolescent Health $(\mathrm{N}=10,076)$.

Study design-Self-reported sleep duration was categorized as $<6,6-8$, or $>8$ hours. Obesity status, using measured height and weight, was defined as Body Mass Index (BMI) $\gg 5^{\text {th }}$ percentile in adolescence and BMI $230 \mathrm{~kg} / \mathrm{m}^{2}$ in young adulthood.

Results-Cross-sectionally, short sleep duration was associated with obesity in adolescent males (Prevalence Ratio (PR) 1.8 [95\% CI: 1.3, 2.4]) but not in females (PR 1.0 [95\% CI: 0.7, 1.4]). In longitudinal analyses, short sleep duration in adolescence was associated with incident obesity in both males and females (Risk Ratio (RR) 1.2 [95\% CI: 1.0, 1.6]) in young adulthood. No interactions by sex were noted.
\end{abstract}

Conclusions-Analyzing the association of sleep duration and obesity longitudinally resolved sex discrepancies observed in earlier cross-sectional analyses. Optimizing sleep duration during adolescence may be an effective intervention to prevent excess weight gain in young adults.

\section{Keywords}

Obesity; sleep; adolescents; sex differences

The high prevalence of obesity among children and adolescents in the US is likely to be multifactorial, [1] including social, environmental and behavioral risk factors. Short sleep duration, a modifiable health behavior, has been hypothesized to affect weight gain through

(C) 2014 Mosby, Inc. All rights reserved.

Corresponding author: Shakira F Suglia, Columbia University Mailman School of Public Health 722 West $168^{\text {th }}$ St, New York NY 10032, Phone 212-342-5241, sfs2150@ columbia.edu.

The authors declare no conflicts of interest.

Publisher's Disclaimer: This is a PDF file of an unedited manuscript that has been accepted for publication. As a service to our customers we are providing this early version of the manuscript. The manuscript will undergo copyediting, typesetting, and review of the resulting proof before it is published in its final citable form. Please note that during the production process errors may be discovered which could affect the content, and all legal disclaimers that apply to the journal pertain. 
disruption of several hormones including leptin, insulin, cortisol, ghrelin and growth hormones [2-5]. Furthermore, daytime sleepiness and fatigue as a result of short sleep duration can alter eating behaviors and physical activity, potentially leading to weight gain [6-8].

An association between short sleep duration and obesity among school age children and preadolescents has been reported [9-12]. In a 2008 meta-analysis children < 10 years of age with short sleep duration had a 58\% higher odds of overweight or obesity compared with children with longer sleep duration [9]. Findings among adolescents are not as clear [13]. Most studies among adolescents have relied on cross-sectional data and have noted significant positive associations between short sleep duration and obesity [14-16]. However, within the National Longitudinal Study of Adolescent Health no significant associations between short sleep duration in adolescence and obesity risk after a one-year follow-up was noted [17, 18]. Although the length of follow-up time was short these contradictory findings raise the possibility that the effects previously noted in cross-sectional studies are attributed to the effects of obesity on sleep duration and not of sleep duration on obesity, as previously thought. As many health behaviors are established in adolescence, it is plausible that sleep duration during the adolescent years is closely related to sleep duration in adulthood, which has been associated with various adult health outcomes, including obesity [19, 20].

Another potential source of discrepancies in results from previous studies of adolescents is sex differences. Differential effects for males and females could lead to an average null effect. Only a few studies have investigated sex differences. A few studies have noted an effect of short sleep duration on childhood obesity only among males [18, 21, 22]. However, one cross-sectional US study noted a positive association between short sleep duration and being overweight among female high school students [23]. Sleep duration has also been negatively associated with BMI among Hispanic, White and Asian adolescent boys and positively associated with Black adolescent girls after a one-year follow-up [24]. The discrepancy in study findings could be due to the cross-sectional nature of these studies or short follow-up period.

We explored both the cross-sectional association between adolescent short sleep duration and obesity, and the longitudinal associations between adolescent short sleep duration and incident obesity during the transition to young adulthood. Given the potential for differential effects of short sleep duration we examine whether the associations differ by sex.

\section{Methods}

The National Longitudinal Study of Adolescent Health (Add Health) study is a nationally representative school-based, longitudinal study of the health-related behaviors of adolescents and their outcomes in young adulthood. An in-school questionnaire was administered to a nationally representative sample of students in grades 7 through 12, plus selected oversampled minority groups, stratified by age and sex, during the 1994-1995 school year in 132 schools. The study design has been described in detail elsewhere [25]. Briefly, 80 high schools representative of US schools were selected with respect to region of country, urbanicity, size, type and ethnicity. Eligible schools included an $11^{\text {th }}$ grade and 
enrolled more than 30 students. The first wave of in-home interviews was conducted among adolescents 12 to 18 years of age between 1994 and 1995 (Wave 1). Wave 2 (1996; mean age 16 years) follow-up was conducted one year after Wave 1, and included 13,570 adolescents who would still be enrolled in high school during 1996, including dropouts, who were part of the nationally representative sample. Wave 3 (2001; mean age 20 years) included 10,393 of those Wave 2 respondents (76.5\%). Self-reported height and weight was used for participants missing measured height and weight at wave $2(\mathrm{~N}=103)$ or wave 3 $(\mathrm{N}=324)$. In these analyses our sample consists of participants with either height and weight measurements or self-reported measures at waves 2 and 3 and complete information on covariates of interest $(\mathrm{N}=10076)$. The Add Health study was approved by the institutional review board of the University of North Carolina, Chapel Hill. Informed consent by participants was provided in written form. The current analyses were approved by the institutional review board of Columbia University, New York.

During wave 2, one year after the baseline examination, home visits were conducted to assess the adolescent's height and weight. Height and weight were measured by trained study staff at the participant's home. Body mass index (BMI) was calculated from measured height and weight $\left(\mathrm{kg} / \mathrm{m}^{2}\right)$ or self-reported height and weight for participants missing measured height and weight at wave $2(\mathrm{~N}=103)$ or wave $3(\mathrm{~N}=324)$. The Center for Disease Control and Prevention (CDC) 2000 BMI growth references [26] were used to determine age- and sex-specific BMI percentiles (BMIp). Obesity during adolescence was defined as BMI $\geq 95^{\text {th }}$ percentile at the Wave 2 assessment. During wave 3 , height and weight were again measured during home visits by trained study staff. Obesity in adulthood was defined as BMI $230 \mathrm{~kg} / \mathrm{m}^{2}$.

At wave 2, adolescents were asked: "How many hours of sleep do you usually get?" The current sleep recommendations for adolescents range between 8 and 9 hours of sleep per night depending on the entity issuing the recommendations [27]. Sleep duration in adolescence was categorized as, less than 6 hours, between 6 and 8 hours, and more than 8 hours of sleep.

Questionnaires ascertained information on socio-demographic factors, including age, sex, race/ethnicity, parental highest education level achieved (wave 2), hours spent watching TV and self-reported leisure-time physical activity. Age was categorized as 11-13, 14-15, 16 or 17 years of age or older. Race/Ethnicity was categorized as white non-Hispanic, African American, Hispanic or other race/ethnic group. Parental education was based on the highest education level attained by either the mother or the father and was classified as: less than high school, high school graduate or vocational school, some college, college graduate or graduate education. Hours per week spent watching television were characterized as more or less than 2 hours of television watching per day. Physical activity was defined as 5 or more bouts of moderate to vigorous physical activity per week, based on self-report at wave 2 .

\section{Data Analyses}

Binomial regression analyses were conducted to estimate the association between adolescent sleep duration at wave 2 and adolescent obesity at wave 2, adjusting for socio-demographic factors (age, education, race/ethnicity). A second model examined the longitudinal 
association between adolescent short sleep duration (wave 2) and young adult incident obesity (wave 3), adjusting for socio-demographic factors. Sex by sleep duration interactions were tested in both models. Additional analysis further adjusted for TV watching and physical activity. All analyses were weighted to account for the complex sampling design and clustering of the data and conducted in SAS version 9.0 (SAS Institute, Cary, NC).

\section{Results}

In the final sample of 10076 participants, 53\% were female, $14 \%$ were African - American and $11 \%$ Hispanic (Table I). The mean age of participants at adolescence was 16 years and the mean age in the adult follow-up was 21 years. In adolescence $14 \%$ of participants were obese. In young adulthood, the prevalence of obesity rose to $21 \%$. Sex differences were noted for health behaviors, sleep duration, and obesity (Table I). Men were more likely to be physically active, more likely to be obese in adolescence, were more likely to sleep less than 6 hours a night in adulthood and were more likely to report watching more than 2 hours of $\mathrm{TV}$ in adolescence, compared with women (all $\mathrm{p}<0.05$ ).

In cross-sectional analyses adjusted for demographic factors, sleeping less than 6 and between 6 and 8 hours per night during adolescence was associated with adolescent obesity among males compared with those sleeping more than 8 hours per night: (prevalence ratio [PR] 1.8 [95\%CI: 1.3, 2.4] and PR 1.3 [95\% CI: 1.0, 1.7], respectively) but not among females (sex interaction $\mathrm{p}$-value $=0.02$ and $\mathrm{p}=0.06$ ) (Table II). Further adjustment for physical activity and time spent watching TV did not appreciably change the estimates.

Table III shows results from analysis of adolescent sleep duration and incident obesity in young adulthood. Short sleep duration, less than 6 hours per night, compared with sleeping more than 8 hours was associated with incident obesity in the full sample (RR $1.295 \% \mathrm{CI}$ $1.0,1.6$ ), this association did not differ by sex (sex interaction $\mathrm{p}$-value $=0.25$ ).

\section{Discussion}

In this nationally representative sample of U.S. adolescents, short sleep duration in adolescence was associated with obesity among boys but not girls. However, when we examined the association between adolescent sleep duration and onset of obesity in young adulthood, a longitudinal association of similar magnitude was noted for both males and females.

A prior analysis of the association between short sleep duration and obesity among adolescents participating in the Add Health study noted no significant association [17]. However, the authors failed to account for the differential associations by sex, which obscured the association between short sleep duration and obesity among males that we noted in our analysis. Knutson examined the cross-sectional association between sleep duration, as a continuous measure, and BMI z-score among adolescents in the public-use version (smaller sample) of the Add Health study, noting a significant association among males only. [18] Instead, we used the national representative sample of the Add Health study and explored longitudinal associations. Other studies have noted sex differences on the association between short sleep duration and obesity.[18, 24] A cohort study of adolescents 
in Porto, Portugal, explored the association between short sleep duration and body fat percentage as well as BMI z-scores among adolescents. [28] Similar to our findings they noted both a cross-sectional (age 13) and longitudinal (age 13 to 17) inverse association between sleep duration and BMI z-score among boys, but no associations were noted among girls. [28] Our longitudinal analysis differs in that we examine the longitudinal associations as adolescents transition to adulthood noting longitudinal associations among both girls and boys. Furthermore in contrast to the Porto study, our analyses follow a nationally representative sample of adolescents.

Longitudinal investigations of incident obesity provide stronger causal evidence than do cross-sectional analysis. Although we note different results for males and females in our cross-sectional analysis, the longitudinal analysis revealed a positive relation between sleep duration and obesity among both sexes. This study provides evidence that there may be a sex-neutral effect of short sleep duration on obesity incidence that is obscured in crosssectional analysis. One explanation for the sex differences in cross-sectional analysis is that cross-sectional analyses can be biased by reverse causation. It is possible that obesity may have differential effects on sleep duration in boys and girls. Perhaps, in boys, the physiological effects of more disordered and shorter duration of sleep, predominate and lead to a stronger association between short sleep duration and obesity. In girls, psychological effects of obesity, including depression and increased sleep duration, may be more pronounced and thereby lead to a weakened association between short sleep duration and obesity.

It is also possible, however, that there are true sex differences in the association between sleep duration and obesity. Sex differences may be attributed to physiological mechanisms. Adolescence is a period of physical development for both men and women, and differential changes in body composition during this period may be obscuring the association between sleep duration and obesity among women. Our analyses examining obesity in young adulthood among women suggest an association between short sleep duration and obesity that was not apparent during adolescence.

This study has a number of strengths. We used a nationally representative sample of US high school adolescents. The ethnic and geographic diversity of this sample increases its relevance and translatability to contemporary US adolescents. Additionally, we assessed obesity using objectively measured BMI to minimize possible measurement bias. Further, we examined the longitudinal association between sleep duration and obesity in addition to the cross-sectional association, addressing a methodological limitation of prior studies. Finally, we took a life course approach, examining long-term effects of adolescent sleep patterns into adulthood.

There are also limitations to the study. Our assessment of sleep duration was based on selfreport. We have no information on the quality of sleep or other indicators of sleep problems among the respondents. Even though we adjusted for potential confounding factors such as physical activity and hours spent watching TV, we have no information on dietary habits. 
In this nationally representative sample of U.S. adolescents, non-obese adolescents who reported sleeping less than 6 hours per night were more likely to become obese by their early 20s than were their non-obese peers who reported sleeping more than 6 hours a night. These results help clarify inconsistencies in previous cross-sectional findings of null associations between short sleep duration and obesity. Moreover, our findings are consistent with other studies demonstrating the long-term effects of adolescent behaviors and experiences on adult health. Optimizing sleep duration during adolescence may be an important modifiable intervention for obesity prevalence in older adolescents and young adults.

\section{Acknowledgments}

We thank the following individuals from Add Health (University of North Carolina at Chapel Hill): Kathleen Mullan Harris (program project director) and J. Richard Udry, Peter S. Bearman, and Kathleen Mullan Harris (designers). We also thank Ronald R. Rindfuss and Barbara Entwisle for assistance in the original design of the study.

S.S. was supported by the National Institutes of Health (NIH; Number K01HL103199). W.R. was supported by NIH (K01CA172717) and the Carolina Population Center (R24 HD050924). This research uses data from Add Health, which is funded by the Eunice Kennedy Shriver National Institute of Child Health and Human Development (P01-HD31921), with cooperative funding from 23 other federal agencies and foundations. No direct support was received from Eunice Kennedy Shriver National Institute of Child Health and Human Development for this analysis.

\section{References}

1. Ogden CL, Carroll MD, Kit BK, Flegal KM. Prevalence of obesity and trends in body mass index among US children and adolescents, 1999-2010. JAMA. 2012; 307:483-90. [PubMed: 22253364]

2. Beebe DW, Simon S, Summer S, Hemmer S, Strotman D, Dolan LM. Dietary intake following experimentally restricted sleep in adolescents. Sleep. 2013; 36:827-34. [PubMed: 23729925]

3. Hogenkamp PS, Nilsson E, Nilsson VC, Chapman CD, Vogel H, Lundberg LS, et al. Acute sleep deprivation increases portion size and affects food choice in young men. Psychoneuroendocrinology. 2013; 38:1668-74. [PubMed: 23428257]

4. St-Onge MP, Shechter A. Sleep restriction in adolescents: forging the path towards obesity and diabetes? Sleep. 2013; 36:813-4. [PubMed: 23729920]

5. Wheaton AG, Perry GS, Chapman DP, Croft JB. Self-reported sleep duration and weight-control strategies among U.S. high school students. Sleep. 2013; 36:1139-45. [PubMed: 23904673]

6. Magee CA, Huang XF, Iverson DC, Caputi P. Examining the pathways linking chronic sleep restriction to obesity. J Obesity. 2010; 2010

7. Spiegel K, Leproult R, Van Cauter E. Impact of sleep debt on metabolic and endocrine function. Lancet. 1999; 354:1435-9. [PubMed: 10543671]

8. Spiegel K, Leproult R, L'Hermite-Baleriaux M, Copinschi G, Penev PD, Van Cauter E. Leptin levels are dependent on sleep duration: relationships with sympathovagal balance, carbohydrate regulation, cortisol, and thyrotropin. J Clin Endocrinol Metab. 2004; 89:5762-71. [PubMed: 15531540]

9. Chen X, Beydoun MA, Wang Y. Is sleep duration associated with childhood obesity? A systematic review and meta-analysis. Obesity. 2008; 16:265-74. [PubMed: 18239632]

10. Seegers V, Petit D, Falissard B, Vitaro F, Tremblay RE, Montplaisir J, et al. Short sleep duration and body mass index: a prospective longitudinal study in preadolescence. Am J Epidemiol. 2011; 173:621-9. [PubMed: 21303806]

11. Magee L, Hale L. Longitudinal associations between sleep duration and subsequent weight gain: a systematic review. Sleep Med Rev. 2012; 16:231-41. [PubMed: 21784678]

12. Patel SR, Hu FB. Short sleep duration and weight gain: a systematic review. Obesity. 2008; 16:643-53. [PubMed: 18239586] 
13. Guidolin M, Gradisar M. Is shortened sleep duration a risk factor for overweight and obesity during adolescence? A review of the empirical literature. Sleep Med. 2012; 13:779-86. [PubMed: 22633283]

14. Knutson KL, Lauderdale DS. Sleep duration and overweight in adolescents: self-reported sleep hours versus time diaries. Pediatrics. 2007; 119:e1056-62. [PubMed: 17473079]

15. Seicean A, Redline S, Seicean S, Kirchner HL, Gao Y, Sekine M, et al. Association between short sleeping hours and overweight in adolescents: results from a US Suburban High School survey. Sleep \& breathing $=$ Schlaf \& Atmung. 2007; 11:285-93. [PubMed: 17440761]

16. Gupta NK, Mueller WH, Chan W, Meininger JC. Is obesity associated with poor sleep quality in adolescents? Am J Hum Biol. 2002; 14:762-8. [PubMed: 12400037]

17. Calamaro CJ, Park S, Mason TB, Marcus CL, Weaver TE, Pack A, et al. Shortened sleep duration does not predict obesity in adolescents. J Sleep Res. 2010; 19:559-66. [PubMed: 20545836]

18. Knutson KL. Sex differences in the association between sleep and body mass index in adolescents. J Pediatr. 2005; 147:830-4. [PubMed: 16356441]

19. Cappuccio FP, Taggart FM, Kandala NB, Currie A, Peile E, Stranges S, et al. Meta-analysis of short sleep duration and obesity in children and adults. Sleep. 2008; 31:619-26. [PubMed: 18517032]

20. Gangwisch JE, Heymsfield SB, Boden-Albala B, Buijs RM, Kreier F, Pickering TG, et al. Short sleep duration as a risk factor for hypertension: analyses of the first National Health and Nutrition Examination Survey. Hypertension. 2006; 47:833-9. [PubMed: 16585410]

21. Eisenmann JC, Ekkekakis P, Holmes M. Sleep duration and overweight among Australian children and adolescents. Acta Paediatr. 2006; 95:956-63. [PubMed: 16882569]

22. Ramos E, Barros H. Family and school determinants of overweight in 13-year-old Portuguese adolescents. Acta Paediatr. 2007; 96:281-6. [PubMed: 17429921]

23. Lytle LA, Pasch KE, Farbakhsh K. The relationship between sleep and weight in a sample of adolescents. Obesity. 2011; 19:324-31. [PubMed: 20948522]

24. Reither EN, Krueger PM, Hale L, Reiter EM, Peppard PE. Ethnic variation in the association between sleep and body mass among US adolescents. Int J Obes. 2014

25. Popkin BM, Udry JR. Adolescent obesity increases significantly in second and third generation U.S. immigrants: the National Longitudinal Study of Adolescent Health. J Nutr. 1998; 128:701-6. [PubMed: 9521631]

26. Statistics NCfH. 2000 CDC Growth Charts: United States. Hyattsville, MD: 2004.

27. Matricciani LA, Olds TS, Blunden S, Rigney G, Williams MT. Never enough sleep: a brief history of sleep recommendations for children. Pediatrics. 2012; 129:548-56. [PubMed: 22331340]

28. Araujo J, Severo M, Ramos E. Sleep duration and adiposity during adolescence. Pediatrics. 2012; 130:e1146-54. [PubMed: 23027175]

\section{Abbreviations}

BMI

Add Health

PR

RR

CI
Body Mass Index

National Longitudinal Study of Adolescent Health

Prevalence Ratio

Risk Ratio

Confidence Interval 
Table 1

Demographics characteristics, sleep duration and obesity, National Longitudinal Study of Adolescent Health $(\mathbf{N}=10076)$

\begin{tabular}{|c|c|c|c|c|}
\hline & \multicolumn{2}{|c|}{$\begin{array}{c}\text { Male } \\
\mathrm{N}=4768\end{array}$} & \multicolumn{2}{|c|}{$\begin{array}{l}\text { Female } \\
\mathbf{N}=5308\end{array}$} \\
\hline & Mean & SE & Mean & SE \\
\hline Age at Wave 2 & 16.0 & 0.03 & 15.8 & 0.03 \\
\hline \multirow[t]{2}{*}{ Age at Wave 3} & 21.5 & 0.03 & 21.2 & 0.03 \\
\hline & Percent & SE & Percent & SE \\
\hline \multicolumn{5}{|l|}{ Race/Ethnicity } \\
\hline White & 67.8 & 0.9 & 68.6 & 0.8 \\
\hline African American & 13.4 & 0.6 & 14.8 & 0.6 \\
\hline Asian & 4.2 & 0.4 & 3.7 & 0.3 \\
\hline Hispanic & 11.4 & 0.6 & 10.4 & 9.5 \\
\hline Other & 4.2 & 0.7 & 3.3 & 0.6 \\
\hline \multicolumn{5}{|l|}{ Parental Highest Education level } \\
\hline Less than high School & 11.4 & 0.6 & 12.1 & 0.6 \\
\hline High School graduate & 31.0 & 0.9 & 31.4 & 0.9 \\
\hline More than High School & 20.7 & 0.8 & 22.4 & 0.8 \\
\hline College Education or Higher & 37.1 & 0.9 & 34.2 & 0.9 \\
\hline TV watching Wave $2>2 \mathrm{hrs} / \mathrm{day}$ & 44.4 & 1.0 & 35.8 & 0.9 \\
\hline TV watching Wave $3>2 \mathrm{hrs} / \mathrm{day}$ & 38.1 & 1.0 & 34.5 & 0.9 \\
\hline Physical Activity Wave 2, 5 + times/wk & 42.5 & 1.0 & 25.4 & 0.8 \\
\hline Physical Activity Wave 3, $5+$ times/wk & 57.1 & 1.0 & 42.4 & 0.9 \\
\hline \multicolumn{5}{|l|}{ Sleep Duration Wave 2 (hrs/night) } \\
\hline Less than 6 hours & 15.7 & 0.7 & 19.5 & 0.7 \\
\hline Between 6 and 8 hours & 61.7 & 1.0 & 60.3 & 0.9 \\
\hline More than 8 hours & 22.7 & 0.9 & 20.1 & 0.9 \\
\hline Obesity Wave 2 & 15.2 & 0.7 & 12.1 & 0.6 \\
\hline Obesity Wave 3 & 21.7 & 0.8 & 23.7 & 0.8 \\
\hline Obesity Wave 3 (incidence) ${ }^{l}$ & 11.5 & 0.7 & 15.0 & 0.7 \\
\hline
\end{tabular}

${ }^{1}$ Sample size $=8718$ 


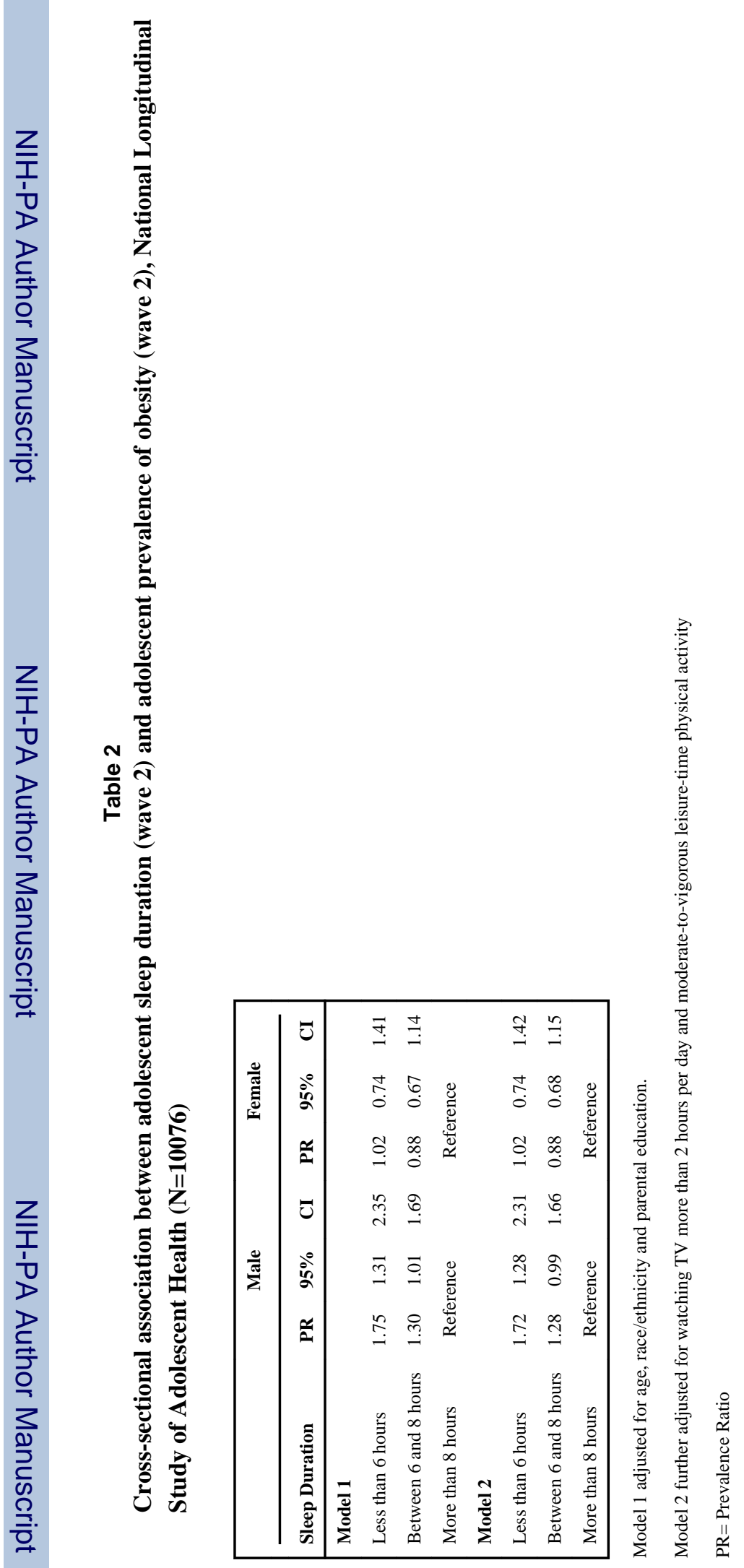

J Pediatr. Author manuscript; available in PMC 2015 October 01. 
Table 3

Longitudinal association between adolescent sleep duration (wave 2) and 5-year incident obesity by young adulthood (wave 3), National Longitudinal Study of Adolescent Health $(\mathrm{N}=\mathbf{8 7 1 8})$

\begin{tabular}{|llll|}
\hline Sleep Duration & RR & 95\% & CI \\
\hline Model 1 & & & \\
Less than 6 hours & 1.24 & 0.99 & 1.56 \\
Between 6 and 8 hours & 1.03 & 0.85 & 1.25 \\
More than 8 hours & Reference & \\
Model 2 & & & \\
Less than 6 hours & 1.25 & 0.99 & 1.41 \\
Between 6 and 8 hours & 1.04 & 0.85 & 1.26 \\
More than 8 hours & Reference & \\
\hline
\end{tabular}

Model 1 adjusted for age, sex, race/ethnicity, and parental education.

Model 2 further adjusted for watching TV more than 2 hours per day and physical activity

$\mathrm{RR}=$ Risk Ratio 